Natural and Applied Sciences International Journal (NASIJ) eISSN: 2788-4619 (Online)

https://doi.org/10.47264/idea.nasij/1.1.5

Vol. 1, No. 1, (January-December 2020), 53-63

https://www.ideapublishers.org/index.php/nasij

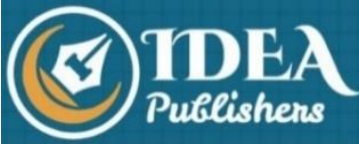

Research Article

\title{
Smart Cloud Based Efficient Doctor Patient Portal of Healthcare System
}

\section{Zahid U. Rahman*}

Department of Computer Science, Comsats University, Islamabad, Pakistan.

* Corresponding Author Email: zahidktk369@gmail.com

\begin{abstract}
Healthcare is one of the most important priority of every country. Most of the developed countries are trying to bring internet to solve health related problems. Meanwhile, healthcare is not that much developed in populated countries like Pakistan. The private health care sector serves more than half of our country's population. We have tried to develop a web based model to transfer the clinical system into the cloud to overcome the doctor burden, and further assist the patients to find a doctor easily. This can save time of both the doctor and patient. The web based patient-doctor database shows credibility in bringing both the ends near and accessible even in low internet connectivity regions. However, there is a back draw to educate the end users about the complexities in the web based doctor portal. This will provide more insights after eliminating health issues and provide an adequate approach to the patients.
\end{abstract}

Keywords: Smart Healthcare; Portal; Doctor; Patient Portal; Online Appointments; Web Based.

\section{How to Cite:}

Rahman, Z. U. (2020). Smart Cloud Based Efficient Doctor Patient Portal of Healthcare System. Natural \& Applied Sciences International Journal (NASIJ), 1(1), 53-63. https://doi.org/10.47264/idea.nasij/1.1.5

\section{Publisher's Note:}

IDEA PUBLISHERS (IDEA Journals Group) stands neutral regarding jurisdictional claims in the published maps and institutional affiliations.

\section{Copyright:}

(C) 2020 The Author(s), published by IDEA PUBLISHERS (IDEA Journals Group)

This is an Open Access article published under the Creative Commons Attribution-NonCommercial 4.0 International License (http://creativecommons.org/licenses/by-nc/4.0/) 


\section{Introduction}

The health care system of Pakistan is mostly promised on private clinics. The private health care sector serves nearly 70 percent of the population according to the World Bank. Every day patients go to the doctors, clinics for their disease checkups. Pakistan is listed as one of the 57 countries with critical health workforce deficiency (Bärnighausen \& Bloom, 2009). Today, doctor to patient ratio in Pakistan is 1:1300 (Pathman et al., 2004; Rana et al., 2016). It means that there is only one doctor available per 1300 people. The connection between a patient and doctor was first presented in Pakistan, which included different facilities; including career development, lack of incentives, quality of life, and lack of connectivity in different regions of Pakistan (Rana et al., 2016). They further illustrate that the rural and urban health facilities, transportation services and governance issues are some of the main factors identified by young doctors in Islamabad Capital Territory that contribute in their decision of choosing a certain job or not in rural areas (Honda \& Vio, 2015).

Moreover, in a clinic system patients contact with the doctors the telephone, or by a person to make an appointment with the doctors (Rao et al., 2013). This process becomes more time consuming and critical to manage on holidays where every patient walks around clinics. Patients wait for their turn in a row in clinics. Doctors check the patients and then write medicines after diagnosing the disease. Sometimes, it becomes more difficult for doctors to manage this system, when the number of patients increases from allotted target on a specific day. Patients suffer from this system too, especially when some of them have serious health issues and they don't get time to meet with doctors on urgent basis. These clinic systems also do not suit to disable persons, old people and children because they can't wait for too long in clinics (Hamouzadeh et al., 2019).

Nowadays, researchers are working to build an online web portal that allow patients to book appointments with doctors via the internet. These systems give an easy way to find out the best doctors and the best treatment, as well as it provides information about the availability of the blood and ambulance system in hospitals. Similarly, they allow patients to check doctor profiles and make appointments with them and a system administrator manages all this process (Pathman et al., 2000). The proposed systems have a lot of facilities, meanwhile they are very complex so that they cannot be managed by doctor or patients. Medical issues using conventional methods also in problems in many countries, specifically in urban regions (Pathman et al., 2000; Rosenthal, 2000). Moreover, the outcomes in web based medical treatment are explained in a precise review to explain the patients and doctor issues in rural, urban and cities of different countries (Aysola et al., 2015).

They surveyed 976 health centres, in which 376 responded. Health centers with career ladder programs compared to those without had higher adjusted rates of no/minimal difficulty in recruitment of primary care providers. (17.6\% vs. $10.6 \%$; $=.01)$ and close to double the adjusted rates of reporting no/minimal difficulty in retention of primary care providers $(39.4 \%$ vs. 21.2\%; p=.0001) (Skar et al., 2015; Walter et al., 2015). The objective of this paper is to identify the best available web based patients and doctor related problem to provide the best health services. Furthermore, the effectiveness of web based family in educational institutes is the first trial basis to run this program. A lot number of patients and doctors will benefit from these services to meet the present day challenges faced both the patients and health staff (Halken, 2004; Mejias \& Ramphul, 2018). 


\section{A. System Design:}

Our system is mainly composed of three parts:

1) Patient Portal

2) Doctor Portal

3) Appointment Portal

We will explain the functions of each of these portals one by one in detail as shown in the Figure-1 below.

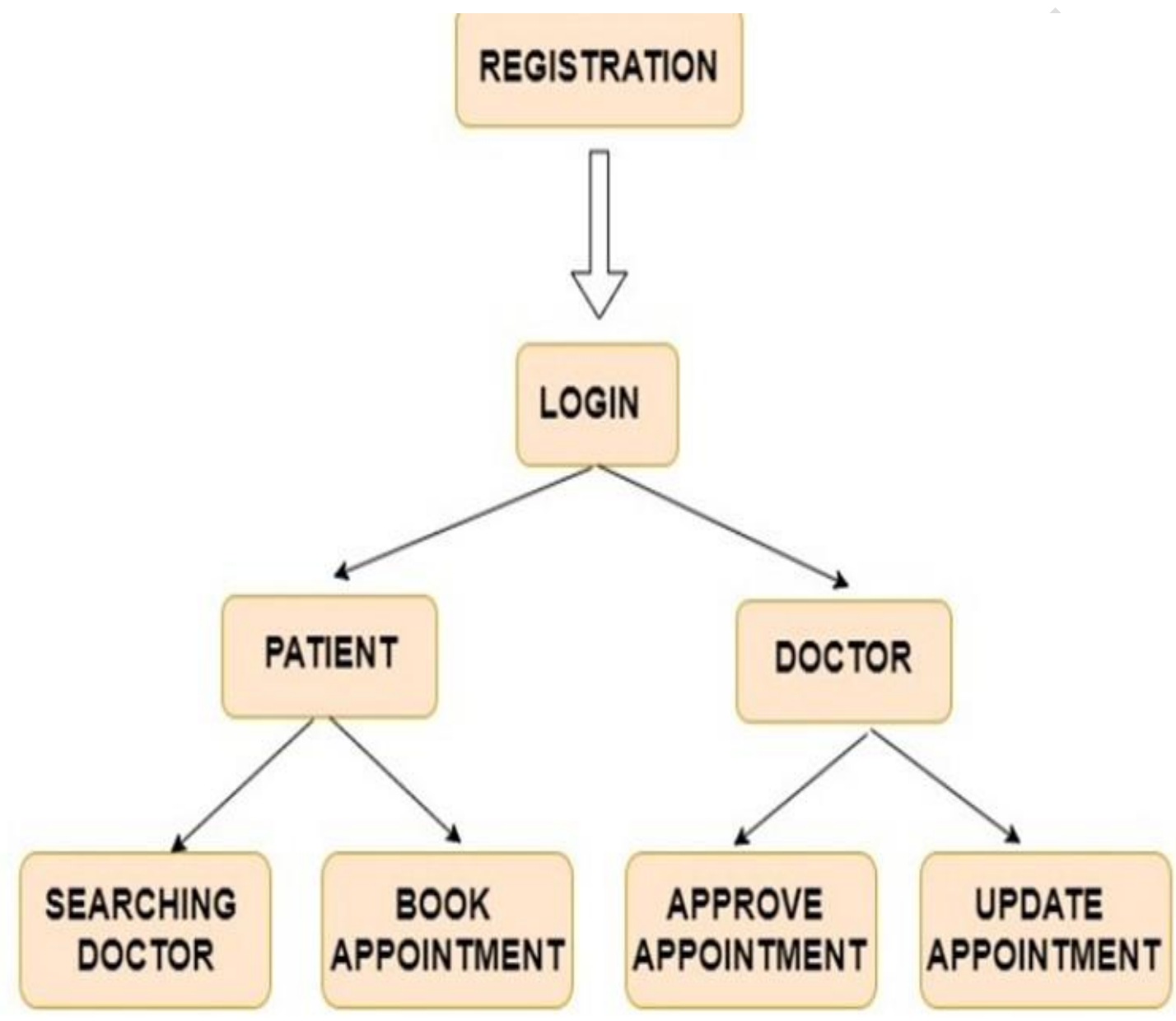

Figure 1: Block diagram

\section{B. Home Page:}

The home page is designed very beautiful and attractive (Figure 2). It contains the registration and login part. There are two options for registration, one for the doctor and another for the patients, which lets them to different registration pages. Similarly, the login pages are designed separately for both the doctor and patient. It means that one for doctors and another for patients. The purpose of designing this page is simplicity to make it understandable for everyone easily. 


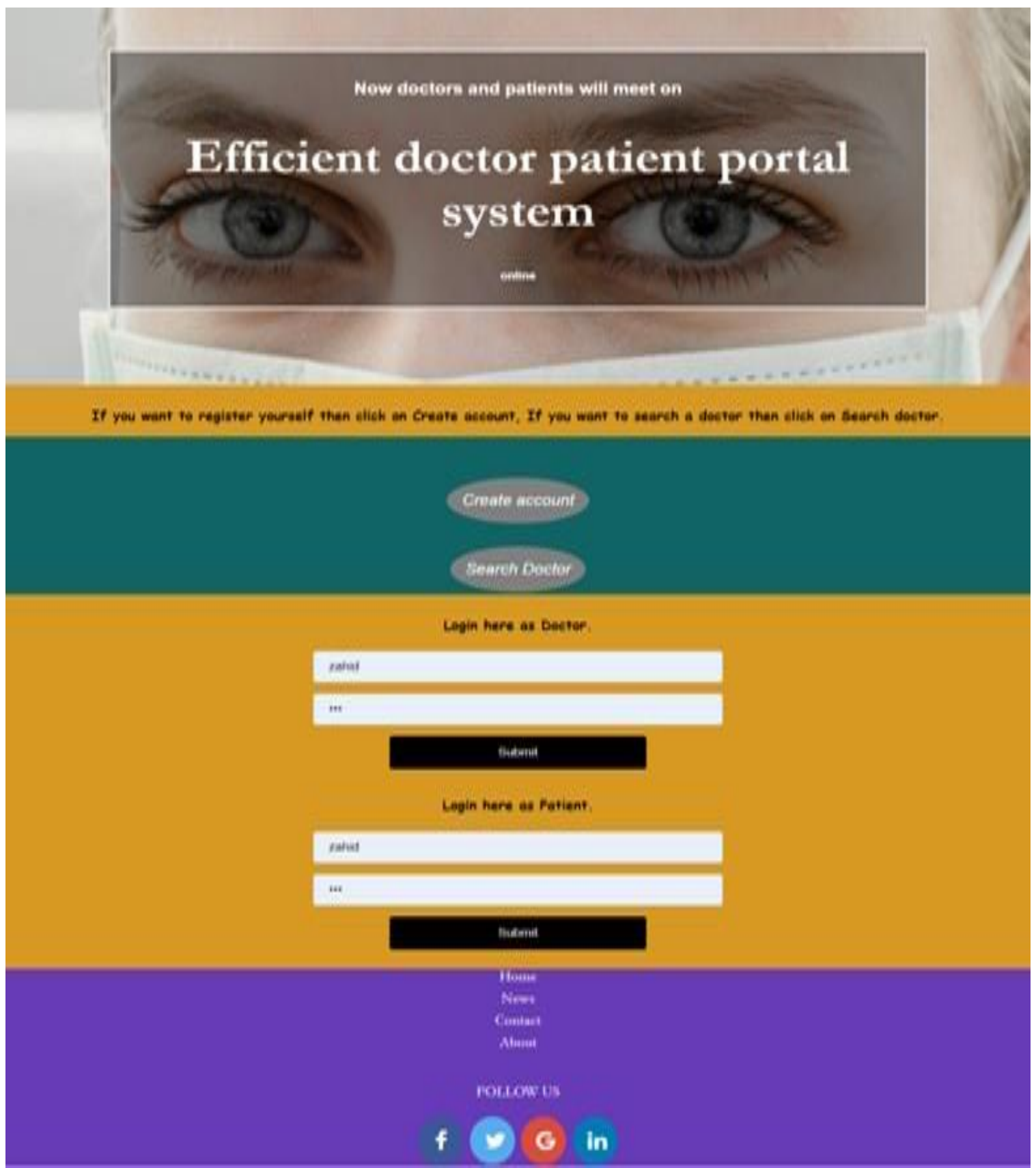

Figure 2: Homepage

\section{Patient Portal:}

This portal displays a registration form to fill the information that uniquely identifies each patient individually (Figure 3). After completion of this form, the patients are registered in the database. Now, they can see their profiles, and moreover, they can update their profiles. They can search doctors and can make appointments with them. They can see the complete detail about their appointment history like how many appointments they have submitted, also the system notifies them with a notification when a doctor makes changes or approve their appointments. 


\section{Fill below form}

\section{Please select check-up date and time}

\section{$8: 00 \mathrm{AM}$}

\section{Submit}

Figure 3: Patient registration page

\section{Doctor Portal:}

This portal allows doctors to make their profiles. After setting their profiles by filling a doctor registration form they are transferred to their profile page where they can update their information record like their clinic timings, leaves and other important information. Each time a doctor wants to access his profile, he needs to log in. The purpose is to make sure the privacy and security of the system that is important to maintain. The doctors can also edit the appointment information submitted by their patients. They set appointment timings for their patients (Figure 4). 


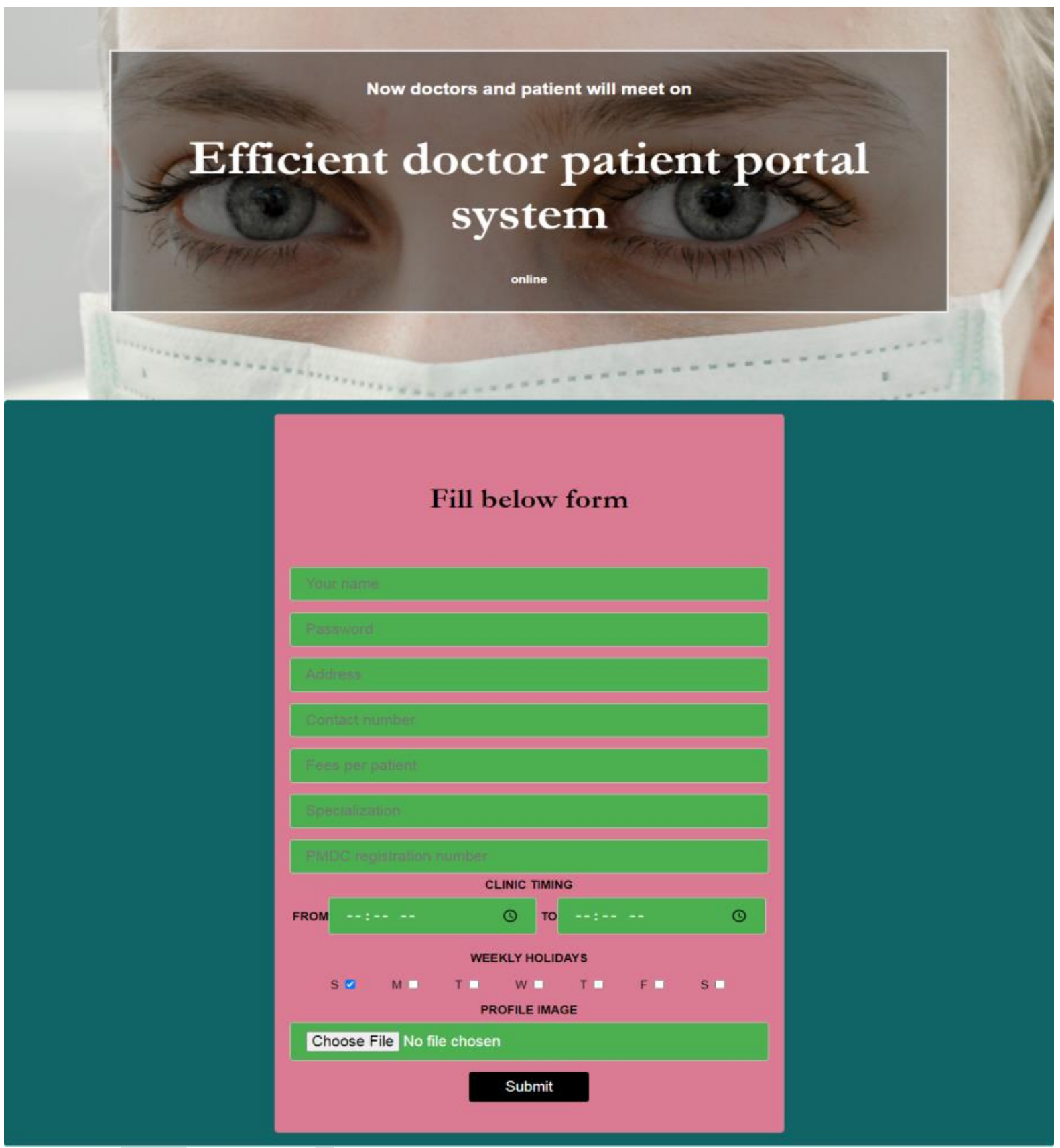

Figure 4: Doctors registration page

\section{E. Appointments Section:}

This is the appointment registration portal, as in Figure 5. Patients fill this form to make an appointment with the doctors. This is very simple. Its database consists of just three fields like patient name, the time and day on which the doctor meetup is scheduled. After submission of this form, it is displayed on the doctor's profile. The doctor checks if he has an available time slot submitted by his patient after that doctor approves the appointment or update the time and date, if he was busy or not prepare to check his patients at that time, mentioned in the appointment by his patient. 


\section{Fill below form}

\section{Please select check-up date and time}

\section{mm/dd/yyyy}

\section{$8: 00 \mathrm{AM}$}

\section{Submit}

Figure 5: Appointment registration page

\section{F. Doctor Details Section:}

This portal lets patients and general users to search and see information about the doctors (Figure 6). They do not need to make a profile or fill any registration form. Its purpose is to attract users to the site and help them to find doctors they need in an emergency. They can search an unlimited number of doctors registered on our site. The page is designed simply which loads fast. 


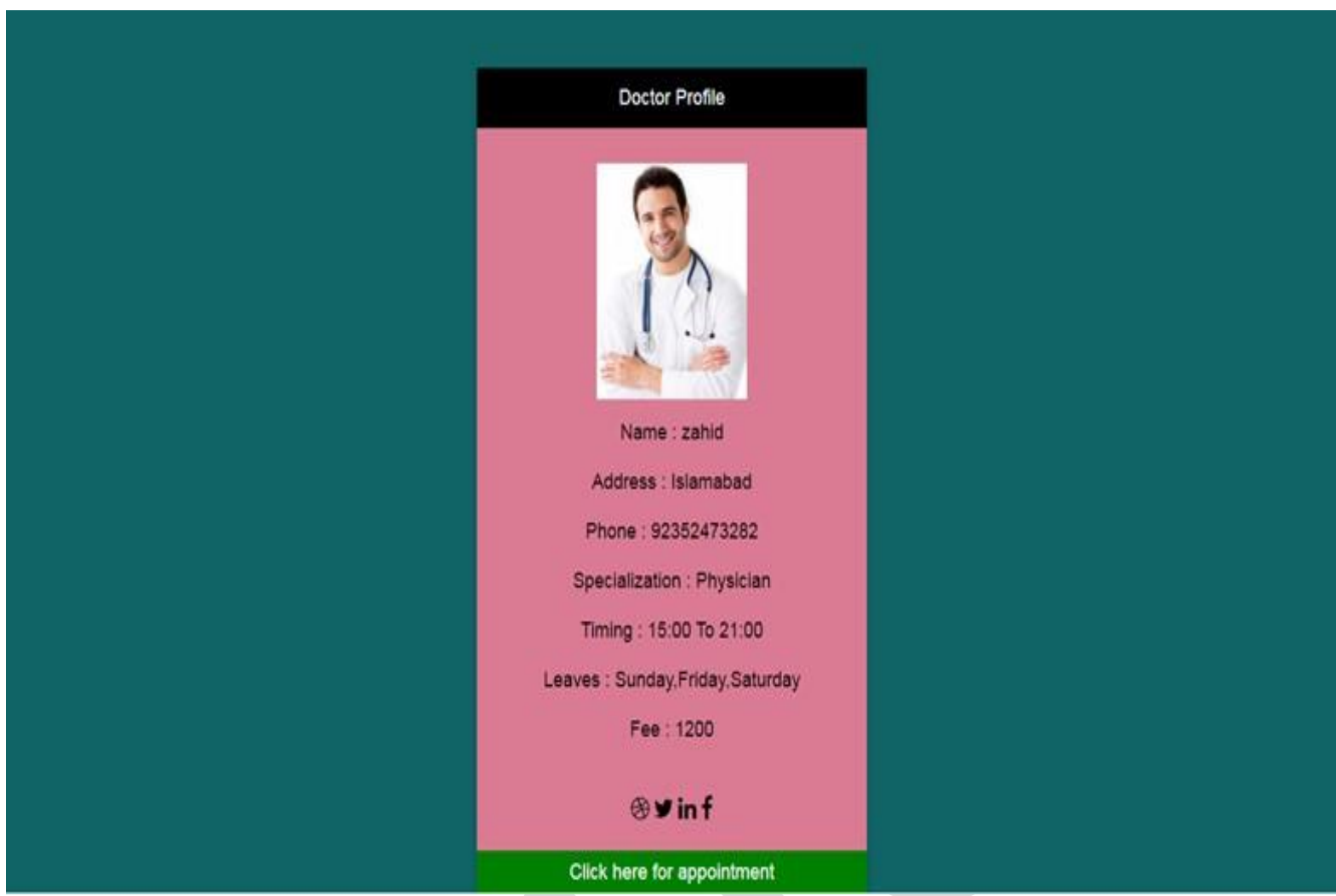

Figure 6: Doctors information page

\section{Database}

As our first priority is to make the simplest model, that anyone can use and understand without any logic. Therefore, we have used just one database in our model consisting of three tables as shown in Figure 2. A. Patient Table This entity stores information about patients. It is consisting of five attributes which is named, password, address, phone number and profile image of the patient as shown in Figure 2.

A. Patient can register appointment for more than one doctor at the same time, but a patient cannot place more than one appointment at the same time for only one doctor. In actual the patient will visit only once to a doctor for a checkup (Figure 7). So to remove redundancy, if a patient mistakenly tries to submit multiple appointment forms for one doctor, he will receive an error message from the system that he has already registered an appointment for this doctor and he can only make another appointment with the doctor if he updates or deletes his first one appointment. Normally updating the old one appointment will be helpful.

B. Appointments Table This table stores the appointment information between a doctor and a patient. It serves as the backbone of this system. It is consists of seven attributes. It comes from the patient side after filling the appointment form (Figure 7). First the patients fill all the attributes and then the doctor make changes. Doctor is able to make changes in date, time and the remarks whether he is available or not. He can also leave remarks attribute empty. Once doctor returns the form it displays back to the patient with a notification on patient portal. 
C. Doctor Table Doctor table stores information about the doctors. It's a little bit longer as compared to a patient table as complete doctor information is important for the success of this system. It consists of nine attributes which provides a detailed overview of the doctor. Some of the most important attributes are clinic timings and specialization. The doctor also needs to provide his registration number, the system will try to match it with the registration number database, if it was available then the system will allow him to create an account. Its purpose is to make sure that each and every doctor is specialized in his field showing on this portal (Figure 8)

\begin{tabular}{|c|c|c|c|c|c|}
\hline \multicolumn{2}{|r|}{ PATIENT } & \multicolumn{2}{|r|}{ APPOINTMENTS } & \multicolumn{2}{|r|}{ DOCTOR } \\
\hline PK & Patient_ID & PK & Appointments_ID & PK & Doctor_ID \\
\hline & $\begin{array}{l}\text { Name } \\
\text { Password } \\
\text { Address } \\
\text { Phone_No } \\
\text { Profle_Picure }\end{array}$ & & $\begin{array}{l}\text { Patient_Name } \\
\text { Doctor_Name } \\
\text { Date } \\
\text { Time_by_Patient } \\
\text { Doctor_Remarks } \\
\text { Patient_ID } \\
\text { Doctor_ID }\end{array}$ & & $\begin{array}{l}\text { Name } \\
\text { Password } \\
\text { Address } \\
\text { Contact } \\
\text { Fee } \\
\text { Specializtion } \\
\text { Clinic_Timing } \\
\text { Registration_Number } \\
\text { Profile_Image }\end{array}$ \\
\hline
\end{tabular}

Figure 7: Database designing in the form of a flowchart

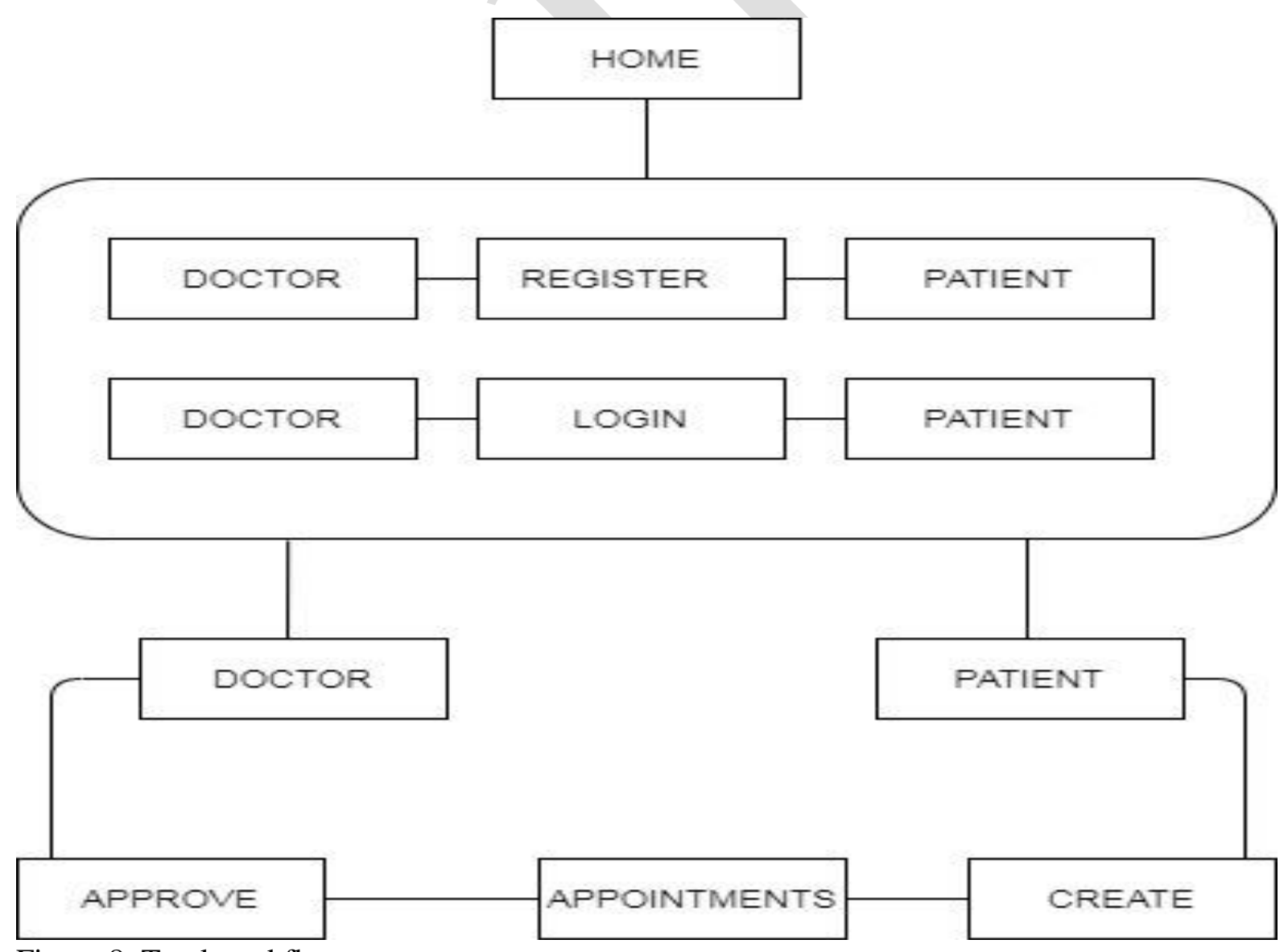

Figure 8: Total workflow 


\section{Conclusion}

In this paper, we presented a cloud based web model allowing doctors and patients to communicate via an efficient doctor-patient portal. The idea is to transfer the conventional clinical systems which doctors use to allocate appointments for their patients. There are many models developed before, but each of them has their own purposes and limitations. Our goal is to make the simplest model that anyone can use and understand without any logic. This system does not need any admin to manage the system. All these things are in the hands of doctors and patients. The system database is consisting of three tables namely doctor, patient and appointment table. The doctor table stores information about the registered doctors. Similarly, the patient table stores information about patients after registrations. The appointment table stores information about the appointments take place between doctors and patients. The future work of this system is to develop a mobile application.

\section{Acknowledgement}

The author is thankful to the editorial board and two anonymous reviewers for their time and valuable comments.

\section{References}

Aysola, J., Groves, D., \& Hicks, L. S. (2015). Health Center Professional Programs and Primary Care Workforce. J Fam Med Community Health, 2(8). https://www.ncbi.nlm.nih.gov/pmc/articles/PMC5120588/

Bärnighausen, T., \& Bloom, D. E. (2009). Financial incentives for return of service in underserved areas: a systematic review. BMC Health Serv Res, 9(1), 1-17. https://doi.org/10.1186/1472-6963-9-86

Halken, S. (2004). Prevention of allergic disease in childhood: clinical and epidemiological aspects of primary and secondary allergy prevention. Pediatr Allergy Immunol, 15 Suppl 16 (4-5), 9-32. DOI:10.1111/j.1399-3038.2004.0148b.x

Hamouzadeh, P., Akbarisari, A., Olyaeemanesh, A., \& Yekaninejad, M. S. (2019). Physician preferences for working in deprived areas: a systematic review of discrete choice experiment. Med J Islam Repub Iran, 33, 83. DOI:10.34171/mjiri.33.83

Honda, A., \& Vio, F. (2015). Incentives for non-physician health professionals to work in the rural and remote areas of Mozambique--a discrete choice experiment for eliciting job preferences. Hum Resour Health, 13, 23. DOI:10.1186/s12960-015-0015-5

Mejias, S. G., \& Ramphul, K. (2018). Prevalence and Associated Risk Factors of Bronchial Asthma in Children in Santo Domingo, Dominican Republic. Cureus, 10(2), e2211. DOI: $10.7759 /$ cureus.2211

Pathman, D. E., Konrad, T. R., King, T. S., Taylor, D. H., Jr., \& Koch, G. G. (2004). Outcomes of states' scholarship, loan repayment, and related programs for physicians. Med Care, 42(6), 560-568. DOI:10.1097/01.mlr.0000128003.81622.ef

Pathman, D. E., Taylor, D. H., Jr., Konrad, T. R., King, T. S., Harris, T., Henderson, T. M., . . . Koch, G. G. (2000). State scholarship, loan forgiveness, and related programs: the unheralded safety net. Jama, 284(16), 2084-2092. DOI:10.1001/jama.284.16.2084

Rana, S. A., Sarfraz, M., Kamran, I., \& Jadoon, H. (2016). Preferences Of Doctors For Working In Rural Islamabad Capital Territory, Pakistan: A Qualitative Study. J Ayub Med Coll 
Abbottabad,

28(3),

591-596.

https://www.jamc.ayubmed.edu.pk/jamc/index.php/jamc/article/view/670/423

Rao, K. D., Ryan, M., Shroff, Z., Vujicic, M., Ramani, S., \& Berman, P. (2013). Rural clinician scarcity and job preferences of doctors and nurses in India: a discrete choice experiment. PLoS ONE, 8(12), e82984. DOI:10.1371/journal.pone.0082984

Rosenthal, T. C. (2000). Outcomes of rural training tracks: a review. J Rural Health, 16(3), 213-216. DOI:10.1111/j.1748-0361.2000.tb00459.x

Skar, P., Young, L., \& Gordon, C. (2015). Changes in blood pressure among users of lay health worker or volunteer operated community-based blood pressure programs over time: a systematic review protocol. JBI Database System Rev Implement Rep, 13(10), 3040. DOI:10.11124/jbisrir-2015-1927

Walter, H., Sadeque-Iqbal, F., Ulysse, R., Castillo, D., Fitzpatrick, A., \& Singleton, J. (2015). The effectiveness of school-based family asthma educational programs on the quality of life and number of asthma exacerbations of children aged five to 18 years diagnosed with asthma: a systematic review protocol. JBI Database System Rev Implement Rep, 13(10), 69-81. DOI:10.11124/jbisrir-2015-2335 